\title{
Invertibility of Nonlinear Differential-Algebraic-Equation Subsystems with Application to Power Systems
}

\author{
Qiang Zang, ${ }^{1,2}$ Kaifeng Zhang, ${ }^{2}$ Xianzhong Dai, ${ }^{2}$ and Ying Zhou ${ }^{3}$ \\ ${ }^{1}$ School of Information and Control Engineering, Nanjing University of Information Science \& Technology, \\ Nanjing 210044, China \\ ${ }^{2}$ Key Laboratory of Measurement and Control of Complex Systems of Engineering, Ministry of Education, Southeast University, \\ Nanjing 210096, China \\ ${ }^{3}$ College of Automation, Nanjing University of Posts and Telecommunications, Nanjing 210003, China
}

Correspondence should be addressed to Qiang Zang; zangq@nuist.edu.cn

Received 28 May 2013; Accepted 7 July 2013

Academic Editor: Baoyong Zhang

Copyright (c) 2013 Qiang Zang et al. This is an open access article distributed under the Creative Commons Attribution License, which permits unrestricted use, distribution, and reproduction in any medium, provided the original work is properly cited.

For nonlinear differential-algebraic-equation subsystems, whose index is one and interconnection input is locally measurable, the problem of invertibility is discussed and the results are applied to the power systems component decentralized control. The inverse systems' definitions for such a class of differential-algebraic-equation subsystems are put forward. A recursive algorithm is proposed to judge whether the controlled systems are invertible. Then physically feasible $\alpha$-order integral right inverse systems are constructed, with which the composite systems are linearizaed and decoupled. Finally, decentralized excitation and valve coordinative control for one synchronous generator within multimachine power systems are studied and the simulation results based on MATLAB demonstrate the effectiveness of the control scheme proposed in this paper.

\section{Introduction}

A number of physical systems such as power systems, economic systems, and constrained robot systems are mathematically described by differential-algebraic-equation (DAE) systems [1]. Various concepts, theories, and methods of ordinary-differential-equation (ODE) systems are extended and great progress has been made for DAE systems [2-6]. In [2], for linear DAE systems with input saturation, the composite nonlinear feedback control problem was considered through introducing the generalized Lyapunov function. In [3], the state-feedback stabilization is considered for nonlinear discrete DAE large-scale control systems using Lyapunov matrix equation. $H_{\infty}$ Observer was designed for a class of continuous time nonlinear DAE systems in [4], where necessary and sufficient condition for observer existence was established under the worst conditions. In [5], the traditional Kalman filter was improved and a recursive state estimation method is presented for nonlinear DAE systems. In [6], the output feedback compensation problem was considered by coupling the design of controller and observer instead of separation principle.

In order to provide a measure of the difference between DAE systems and ODE systems, the notion of differential index is commonly used, which corresponds to the minimum number of differentiations of the algebraic equations required to obtain equivalent ODE systems [7]. Among nonlinear DAE systems, the systems of index one represent an important class of physical systems such as power systems and electric circuits. In [8], the Lyapunov method of nonlinear ODE systems was extended to nonlinear DAE systems of index one and the sufficient conditions of stability are presented. In [9], still for such a class of nonlinear DAE systems, an explicit constructing method was given and the state space orderreduction realization was achieved with application to power systems control.

In most existing results, the controlled systems are treated as isolated ones. However, in many practical applications, controlled systems are subsystems within large-scale systems and there exist mutual influence and constraint between the 
controlled systems and the rest of the large-scale systems. Typically, a so-called "power systems component structural model" put forward in $[10,11]$ for power systems component decentralized control problem just falls into this category. At the same time, it should be noted that DAE subsystems control problems are common not only for power systems but also for large-scale systems of others areas [12]. As far as the authors know, the research about nonlinear DAE subsystems is seldom found.

Among various nonlinear control methods, the invertibility of systems plays an important role in linearization and decoupling of general nonlinear systems (not restricted to affine form) [13]. The research on invertibility of nonlinear DAE systems can be traced to $[14,15]$, where the invertibility of continuous and discrete DAE systems was discussed, respectively.

In this paper, for nonlinear DAE systems whose index is one and interconnection inputs are locally measurable and bounded, the invertibility is studied and the results are applied to power systems component decentralized control. The structure of this paper is as follows. Firstly, the definitions of unit right inverse systems and $\alpha$-order integral right inverse systems are given. Secondly, a recursive algorithm is proposed with which to determine whether the controlled nonlinear DAE subsystems are invertible. If the controlled systems are invertible, physically realizable $\alpha$-order integral right inverse systems are constructed through state-feedback and dynamic compensation, with which the decoupling and linearization of the composite systems are both achieved so that various linear control methods and theories can be applied. At last, decentralized excitation and valve coordinative controller are designed for one synchronous generator set within multimachine power systems. The simulation is conducted based on MATLAB and the simulation results illustrate the effectiveness of the proposed scheme in this paper.

\section{System Description and Problem Formulation}

We consider general nonlinear DAE subsystems as follows:

$$
\begin{gathered}
\dot{x}_{i}=f_{i}\left(x_{i}, z_{i}, u_{i}, \bar{v}_{i}\right), \\
g_{i}\left(x_{i}, z_{i}, u_{i}, \bar{v}_{i}\right)=0, \\
y_{i}=h_{i}\left(x_{i}, z_{i}, u_{i}, \bar{v}_{i}\right), \quad i=1, \ldots, N,
\end{gathered}
$$

where $x_{i} \in N_{0}^{i} \subset R^{n_{i}}, z_{i} \in M_{0}^{i} \subset R^{l_{i}}, u_{i} \in L_{0}^{i} \subset$ $R^{m_{i}}, y_{i} \in K_{0}^{i} \subset R^{m_{i}}, \bar{v}_{i} \in S_{0}^{i} \subset R^{s_{i}}$ are differential variable, algebraic variable, manipulated input, controlled output, and interconnection input, respectively. $\bar{v}_{i}$ reflects the influence of the rest of the large-scale systems on controlled systems (1). $f_{i} \in R^{n_{i}}, g_{i} \in R^{l_{i}}, h_{i} \in R^{m_{i}}$ are smooth vector fields. For the sake of simplicity, we will omit the subscript $i$ of (1) in the remainder of this paper. Let $X_{0}=\left(x_{0}, u_{0}, z_{0}, y_{0}, \bar{v}_{0}\right) \in U_{0}$ is compatible initial conditions, that is, $g\left(x_{0}, z_{0}, u_{0}, \bar{v}_{0}\right)=0$ where $U_{0}=\left[N_{0}, M_{0}, L_{0}, K_{0}, S_{0}\right][8]$.
Throughout this paper, the following basic hypotheses are made for (1).

(H1) The Jacobian matrix of $g(x, z, u, \bar{v})$ with respect to $z$ has constant full rank on $U_{0}$ :

$$
\operatorname{rank}\left(\frac{\partial g}{\partial z}\right)=l, \quad \forall(x, z, u, y, \bar{v}) \in U_{0}
$$

that is, (1) is of index one.

(H2) The interconnection input $\bar{v}$ and its sufficient order derivatives are locally measurable and bounded.

Remark 1. Above basic hypotheses hold for power systems components under normal operating conditions.

In sequel we will give inverse systems definition of nonlinear DAE subsystems (1), including unit right inverse systems and $\alpha$-order integral right inverse systems.

The controlled output $y(t)$ of nonlinear DAE subsystems (1) is determined together by the interconnection input $\bar{v}$, manipulated input $u(t)$ and initial conditions. From the functional point of view, nonlinear DAE subsystems (1) can be regarded as an operator (marked by $\theta$ ) which maps the manipulated input $u(t)$, and interconnection input $\bar{v}(t)$ to control output $y(t)$, that is,

$$
y=\theta(u, \bar{v})
$$

Definition 2. Suppose there exist systems $\bar{\Sigma}$ which bear inputoutput mapping relationship: $\hat{y}=\bar{\theta}(r, \bar{v})$, where the input $r(t)=\left(r_{1}, \ldots, r_{m}\right)^{T}$ is a smooth vector. For nonlinear DAE subsystems (1), if $u(t)=\widehat{y}(t)$, we have $y(t)=r(t)$, and then the systems $\Sigma$ are the unit right inverse systems of nonlinear DAE subsystems (1). We call systems (1) are invertible.

Definition 3. Suppose there exist systems $\bar{\Sigma}_{\alpha}$ which bear input-output mapping relationship: $\hat{y}=\bar{\theta}_{\alpha}(\varphi, \bar{v})$ where the input $\varphi(t)=\left(\varphi_{1}, \ldots, \varphi_{m}\right)^{T}=r^{(\alpha)}=\left(r_{1}^{\left(\alpha_{1}\right)}, \ldots, r_{m}^{\left(\alpha_{m}\right)}\right)^{T}$ is a continuous vector. For nonlinear DAE subsystems (1), if $u(t)=\widehat{y}(t)$, we have $y^{(\alpha)}=\varphi$ where $y^{(\alpha)}=\left(y_{1}^{\left(\alpha_{1}\right)}, \ldots, y_{m}^{\left(\alpha_{m}\right)}\right)^{T}$ (i.e., $y_{i}^{\left(\alpha_{i}\right)}=\varphi_{i}, i=1, \ldots, m$ ), and then the systems $\sum$ are the unit right inverse systems of nonlinear DAE subsystems (1). We call systems (1) are invertible.

The aim of this paper is to study the invertibility of nonlinear DAE subsystems (1) satisfying (H1) and (H2). The linearization and decoupling of the composite systems can be achieved if the controlled systems (1) are invertible. As a result, various linear control theorems and methods can be applied.

\section{Recursive Algorithm and Invertibility of Nonlinear DAE Subsystems}

We will give a recursive algorithm, with which to determine the invertibility of nonlinear DAE subsystems (1). 
From (2) we know that $(\partial g / \partial z)^{-1}$ exist on $U_{0}$. We use the following operator $E_{\xi}(\cdot)$ :

$$
\left.E_{\xi}(F) \triangleq \frac{\partial F}{\partial \xi}\right|_{0=g(x, z, u, \bar{v})}=\frac{\partial F}{\partial \xi}-\frac{\partial F}{\partial z}\left(\frac{\partial g}{\partial z}\right)^{-1} \frac{\partial g}{\partial \xi}
$$

to denote the Jacobian matrix of vector function $F\left(x, z, u, y, \ldots, y^{(k)}, \bar{v}, \ldots, \bar{v}^{(k)}\right)$ with respect to some variable $\xi \in(x, u, \bar{v})$ under the algebraic constraint $0=g(x, z, u, \bar{v})$. For the limit of space, only the procedure of step $k$ is presented.

Step $k(k=1,2, \ldots)$. Suppose that, until to step $k$, we can get a sequence of nonnegative integers $\gamma_{0}, \ldots, \gamma_{k-2}, \gamma_{k-1}$ and the distribution $\Delta_{k-1}$ with $X_{0}^{k-1}$ is the regular point. Around $X_{0}^{k-1}$ we have $\operatorname{rank} E_{u}\left(h_{k-1}\right)=\gamma_{k-1}$ where $h_{k-1}=\left[\begin{array}{l}H_{k-2} \\ \widehat{h}_{k-1}\end{array}\right]$. Obviously $\gamma_{k-1} \geq \gamma_{k-2}$, and let $\widehat{h}_{k-1,1}$ denote $\gamma_{k-1}-\gamma_{k-2}$ rows chosen from $\widehat{h}_{k-1}$ such that $\operatorname{rank} E_{u}\left(\left[\begin{array}{c}H_{k-2} \\ \hat{h}_{k-1,1}\end{array}\right]\right)=\gamma_{k-1}$. Let $H_{k-1}=\left[\begin{array}{c}H_{k-2} \\ \widehat{h}_{k-1,1}\end{array}\right]$ and $\widehat{h}_{k-1,2}$ denote the rest rows of $\widehat{h}_{k-1}$. Then there exist neighborhood $\bar{U}_{k}=\left[N_{k}, M_{k}, L_{k}, \bar{K}_{k}, \bar{S}_{k}\right] \subseteq U_{k-1}$ and smooth mapping $\lambda_{k-1}\left(x, z, u, y, \ldots, y^{(k-1)}, \bar{v}, \ldots, \bar{v}^{(k-1)}\right)$ such that

$$
E_{u}\left(\widehat{h}_{k-1,2}\right)=\lambda_{k-1}(\cdot) E_{u}\left(H_{k-1}\right) \text {, }
$$

where

$$
\begin{aligned}
N_{k}=\left\{x \mid x \in N_{k-1}, \operatorname{rank}\left(E_{u}\left(h_{k-1}\right)\right)=\gamma_{k-1}\right\} \\
M_{k}=\left\{z \mid z \in M_{k-1}, \operatorname{rank}\left(E_{u}\left(h_{k-1}\right)\right)=\gamma_{k-1}\right\} \\
L_{k}=\left\{u \mid u \in L_{k-1}, \operatorname{rank}\left(E_{u}\left(h_{k-1}\right)\right)=\gamma_{k-1}\right\} \\
\bar{K}_{k}=\left\{\left(y, \ldots, y^{(k-1)}\right) \mid\left(y, \ldots, y^{(k-1)}\right) \in K_{k-1},\right. \\
\\
\left.\operatorname{rank}\left(E_{u}\left(h_{k-1}\right)\right)=\gamma_{k-1}\right\} \\
\bar{S}_{k}=\left\{\left(\bar{v}, \ldots, \bar{v}^{(k-1)}\right) \mid\left(\bar{v}, \ldots, \bar{v}^{(k-1)}\right) \in S_{k-1},\right. \\
\left.\operatorname{rank}\left(E_{u}\left(h_{k-1}\right)\right)=\gamma_{k-1}\right\} .
\end{aligned}
$$

Remark 4. If $E_{u}(h k-1,2)=0$, then we only need to set $\lambda_{k-1}(\cdot)=0$.

Let

$$
\begin{aligned}
\widehat{h}_{k}=\widehat{h}_{k}\left(x, z, u, y, \ldots, y^{(k)}, \bar{v}, \ldots, \bar{v}^{(k)}\right) \\
=\left[E_{x}\left(\widehat{h}_{k-1,2}\right)-\lambda_{k-1}(\cdot) E_{x}\left(H_{k-1}\right)\right] \dot{x} \\
+\sum_{i=0}^{k-1}\left[\frac{\partial \widehat{h}_{k-1,2}}{\partial y^{(i)}}-\lambda_{k-1}(\cdot) \frac{\partial H_{k-1}}{\partial y^{(i)}}\right] y^{(i+1)} \\
+\left[E_{\bar{v}}\left(\widehat{h}_{k-1,2}\right)-\lambda_{k-1}(\cdot) E_{\bar{v}}\left(H_{k-1}\right)\right] \dot{\bar{v}} \\
+\sum_{i=1}^{k-1}\left[\frac{\partial \widehat{h}_{k-1,2}}{\partial \bar{v}^{(i)}}-\lambda_{k-1}(\cdot) \frac{\partial H_{k-1}}{\partial \bar{v}^{(i)}}\right] \bar{v}^{(i+1)}
\end{aligned}
$$

and define recursively

$$
h_{k}\left(x, z, u, y, \ldots, y^{(k)}, \bar{v}, \ldots, \bar{v}^{(k)}\right)=\left[\begin{array}{c}
H_{k-1} \\
\widehat{h}_{k}
\end{array}\right]=0 .
$$

Let $U_{k}=\left[N_{k}, M_{k}, L_{k}, K_{k}, S_{k}\right]$, where $K_{k}=\left[\bar{K}_{k}, \widehat{K}_{k}\right], S_{k}=$ $\left[\bar{S}_{k}, \widehat{S}_{k}\right]$, and $\widehat{K}_{k}, \widehat{S}_{k}$ are the value region of $y^{(k)}$ and $\bar{v}^{(k)}$, respectively, when $x \in N_{k-1}, z \in M_{k-1}, u \in L_{k-1},\left(y, \ldots, y^{(k-1)}\right) \in$ $\bar{K}_{k-1},\left(\bar{v}, \ldots, \bar{v}^{(k-1)}\right) \in \bar{S}_{k-1}$. Accordingly we get the point $X_{0}^{k}=\left(x_{0}, z_{0}, u_{0}, y_{0}, \ldots, y_{0}^{(k)}, \bar{v}_{0}, \ldots, \bar{v}_{0}^{(k)}\right)$ and let $\Delta_{k}$ denote the distribution generated by the row vectors of $E_{u}\left(h_{k}\right)$. If $X_{0}^{k}$ is the regular point of $\Delta_{k}$, that is, the matrix $E_{u}\left(h_{k}\right)$ has constant rank $\gamma_{k}$ at some neighborhood of $X_{0}^{k}$. If $\gamma_{k}=m$, then algorithm stops; if $\gamma_{k}<m$, then algorithm enters next step.

In the above construction we produce a sequence of nonnegative integers $\gamma_{1}, \gamma_{2}, \ldots$ which satisfy $0 \leq \gamma_{1} \leq \gamma_{2} \leq$ $\gamma_{3} \leq \cdots \leq m$.

Definition 5. The relative order $\rho$ for the nonlinear DAE subsystems (1) is the least positive integer $k$ such that $\gamma_{k}=m$ or $\rho=\infty$ if $\gamma_{k}<m$ for all $k=1,2, \ldots$.

The main results of this paper can be concluded into the following theorems.

Theorem 6. Consider the nonlinear DAE subsystems (1) with relative order $\theta$. If $\theta<\infty$, then there exist unit right inverse systems of nonlinear DAE subsystems (1).

Proof. According to the recursive algorithm, at step $\theta$ we can get the following equation:

$$
h_{\theta}\left(x, z, u, y, \ldots, y^{(\theta)}, \bar{v}, \ldots, \bar{v}^{(\theta)}\right)=0
$$

with $\operatorname{rank}\left(E_{u}\left(h_{\theta}\right)\right)=\gamma_{\theta}=m$. By the virtue of the Implicit Function Theorem, (9) determines a unique solution about $u$ :

$$
u=h_{\theta}^{-1}\left(x, z, y, \ldots, y^{(\theta)}, \bar{v}, \ldots, \bar{v}^{(\theta)}\right) .
$$

Denote $\bar{v}_{k}=\left(\bar{v}^{T}, \ldots,\left(\bar{v}^{(k)}\right)^{T}\right)^{T}, k=0,1, \ldots, \theta$ and construct the following systems:

$$
\begin{gathered}
\dot{\hat{x}}=f\left(\widehat{x}, \widehat{z}, h_{\theta}^{-1}\left(\widehat{x}, \widehat{z}, r, \ldots, r^{(\theta)}, \bar{v}_{\theta}\right), \bar{v}\right), \\
\left(\widehat{x}\left(t_{0}\right), \widehat{z}\left(t_{0}\right)\right)=\left(x\left(t_{0}\right), z\left(t_{0}\right)\right) \\
0=g\left(\widehat{x}, \widehat{z}, h_{\theta}^{-1}\left(\widehat{x}, \widehat{z}, r, \ldots, r^{(\theta)}, \bar{v}_{\theta}\right), \bar{v}\right), \\
\hat{y}=h_{\theta}^{-1}\left(\widehat{x}, \widehat{z}, r, \ldots, r^{(\theta)}, \bar{v}_{\theta}\right),
\end{gathered}
$$

where $\hat{x} \in R^{n}, \widehat{z} \in R^{l}, r \in R^{m}$ and $\hat{y} \in R^{m}$, are differential variables, algebraic variables, manipulated input, and controlled output, respectively. Systems (11) are unit right inverse systems of nonlinear DAE subsystems (1), and conclusion can be proved as follows.

By virtue of the recursive algorithm, there exists a row transmit matrix $\Pi_{k}=\left[\begin{array}{cc}I_{r_{k-1}} 0 \\ 0 & \widehat{\Pi}_{k 1} \\ 0 & \widehat{\Pi}_{k 2}\end{array}\right], k=1, \ldots, \theta-1$ where 
$\widehat{\Pi}_{k 1} \in R^{\left(\gamma_{k}-\gamma_{k-1}\right) \times\left(m-\gamma_{k-1}\right)}$ and $\widehat{\Pi}_{k 2} \in R^{\left(m-\gamma_{k}\right) \times\left(m-\gamma_{k-1}\right)}$ such that $\left[\begin{array}{c}H_{k-1} \\ \hat{h}_{k, 1}\end{array}\right]=\Pi_{k} \cdot h_{k}$, that is,

$$
\begin{gathered}
H_{k}=\left[\begin{array}{c}
H_{k-1} \\
\widehat{h}_{k, 1}
\end{array}\right]=\left[\widehat{\Pi}_{k 1}\left(\frac{d}{d t} \widehat{h}_{k-1,2}-\lambda_{k-1} \frac{d}{d t} H_{k-1}\right)\right], \\
\widehat{h}_{k, 2}=\widehat{\Pi}_{k 2}\left(\frac{d}{d t} \widehat{h}_{k-1,2}-\lambda_{k} \frac{d}{d t} H_{k-1}\right) .
\end{gathered}
$$

For $h_{0}$ especially, we have $\left[\begin{array}{l}H_{0} \\ \widehat{h}_{0}\end{array}\right]=\left[\begin{array}{l}\widehat{\Pi}_{01} \\ \widehat{\Pi}_{02}\end{array}\right] h_{0}$, where $\widehat{\Pi}_{01} \in$ $R^{r_{0} \times m}$ and $\widehat{\Pi}_{k 2} \in R^{\left(m-\gamma_{0}\right) \times m}$.

When the manipulated input of nonlinear DAE subsystems (1) is set to $u(t)=\widehat{y}(t)$, the states $(x(t), z(t))$ satisfy

$$
\begin{aligned}
\dot{x} & =f(x, z, \hat{y}, \bar{v}), \\
0 & =g(x, z, \widehat{y}, \bar{v}) .
\end{aligned}
$$

From (11) we have $\dot{\hat{x}}=f(\widehat{x}, \widehat{z}, \hat{y}, \bar{v}), 0=g(\hat{x}, \widehat{z}, \hat{y}, \bar{v})$ as well as $\left(\widehat{x}\left(t_{0}\right), \widehat{z}\left(t_{0}\right)\right)=\left(x\left(t_{0}\right), z\left(t_{0}\right)\right)$; thus, we have $(\widehat{x}, \widehat{z})=(x, z)$.

Define $\bar{y}_{0}=\widehat{\Pi}_{0,1} y, \widehat{y}_{0}=\widehat{\Pi}_{0,2} y$ and let $\bar{y}_{k}=$ $\widehat{\Pi}_{k, 1} \widehat{y}_{k-1}, \widehat{y}_{k}=\widehat{\Pi}_{k, 2} \widehat{y}_{k-1}, k=1, \ldots, \theta-1$. It can be proved that if the manipulated input $r(t) \in C^{n}(R)$ of systems (11) satisfies $h_{\alpha}\left(\widehat{x}, \widehat{z}, \widehat{y}, r, \ldots, r^{(\alpha)}, \bar{v}_{\theta}\right)=0$ and

$$
\begin{aligned}
& H_{k}\left(x, z, u, r, \ldots, r^{(k)}, \bar{v}_{k}\right) \\
& \quad=H_{k}\left(x, z, u, y, \ldots, y^{(k)}, \bar{v}_{k}\right), \quad k=1, \ldots, \theta-1
\end{aligned}
$$

as well as initial conditions satisfying

$$
\begin{gathered}
\widehat{h}_{k, 2}\left(x\left(t_{0}\right), z\left(t_{0}\right), u\left(t_{0}\right), r\left(t_{0}\right), \ldots, r^{(k)}\left(t_{0}\right), \bar{v}_{k}\left(t_{0}\right)\right) \\
=\widehat{h}_{k, 2}\left(x\left(t_{0}\right), z\left(t_{0}\right), u\left(t_{0}\right), y\left(t_{0}\right), \ldots,\right. \\
\left.y^{(k)}\left(t_{0}\right), \bar{v}_{k}\left(t_{0}\right)\right),
\end{gathered}
$$

then we have $y(t)=r(t)$ where $y(t)$ is the controlled output of nonlinear DAE subsystems (1). From Definition 2, systems (11) are unit right inverse systems of nonlinear DAE subsystems (1). This completes the proof.

Since $(\widehat{x}, \widehat{z})=(x, z)$, unit right inverse systems (11) can be realized by directly feedbacking states $(x, z)$ of nonlinear DAE subsystems (1). The composite systems will be linearized and decoupled when (11) is series connected before (1).

It should be noted that there exist differential operator in above realization (11), which is difficult to be realized in physics. For this we have the following theorem.

Theorem 7. Consider the nonlinear DAE subsystems (1) with relative $\operatorname{order} \theta$. If $\theta<\infty$, then there exist $\alpha$-order integral right inverse systems of nonlinear DAE subsystems (1).

Proof. Replace $y$ of (10) with $r$ :

$$
u=h_{\theta}^{-1}\left(x, z, r, \ldots, r^{(\theta)}, \bar{v}, \ldots, \bar{v}^{(\theta)}\right) .
$$

Let the highest and lowest order derivative of $r_{i}(i=1, \ldots, m)$ are $r_{i}^{\left(\beta_{i}\right)}$ and $r_{i}^{\left(\alpha_{i}\right)}$, respectively. Define

$$
\begin{gathered}
\varphi=\left(\varphi_{1}, \ldots, \varphi_{m}\right)^{T}=\left(r_{1}^{\left(\alpha_{1}\right)}, \ldots, r_{m}^{\left(\alpha_{m}\right)}\right)^{T}, \\
\xi_{i}=\left(r_{i}^{\left(\beta_{i}\right)}, r_{i}^{\left(\beta_{i}+1\right)}, \ldots, r_{i}^{\left(\alpha_{i}-1\right)}\right)^{T}, \quad i=1, \ldots, m
\end{gathered}
$$

and construct the following systems $\bar{\Sigma}_{\alpha}$ :

$$
\begin{gathered}
\dot{\xi}_{i}=A_{i} \xi_{i}+B_{i} \varphi_{i}, \quad i=1, \ldots, m \\
\widehat{y}=h_{\alpha}^{-1}\left(x, z, \xi, \varphi, \bar{v}_{\alpha}\right),
\end{gathered}
$$

where

$$
A_{i}=\left(\begin{array}{cccc}
0 & 1 & & \\
& \ddots & \ddots & \\
& & \ddots & 1 \\
& & & 0
\end{array}\right)_{\left(\alpha_{i}-\beta_{i}\right) \times\left(\alpha_{i}-\beta_{i}\right)}
$$

$$
\begin{array}{r}
B_{i}=\left(\begin{array}{c}
0 \\
\vdots \\
0 \\
1
\end{array}\right)_{\left(\alpha_{i}-\beta_{i}\right) \times 1}, \\
\xi=\left(\xi_{1}^{T}, \ldots, \xi_{m}^{T}\right)^{T}, \quad \bar{v}_{\theta}=\left(\bar{v}^{T}, \ldots,\left(\bar{v}^{(\theta)}\right)^{T}\right)^{T} .
\end{array}
$$

If $r(t) \in C^{\theta}(R)$ of (18) satisfies

$$
\begin{gathered}
h_{\theta}\left(x, z, u, r, \ldots, r^{(\theta)}, \bar{v}_{\theta}\right)=0, \\
H_{k}\left(x, z, u, r, \ldots, r^{(k)}, \bar{v}_{k}\right) \\
\quad=H_{k}\left(x, z, u, y, \ldots, y^{(k)}, \bar{v}_{k}\right), \quad k=1, \ldots, \theta-1 .
\end{gathered}
$$

Meanwhile initial conditions satisfy

$$
\begin{gathered}
\widehat{h}_{k, 2}\left(x\left(t_{0}\right), z\left(t_{0}\right), u\left(t_{0}\right), r\left(t_{0}\right), \ldots, r^{(k)}\left(t_{0}\right), \bar{v}_{k}\left(t_{0}\right)\right) \\
=\widehat{h}_{k, 2}\left(x\left(t_{0}\right), z\left(t_{0}\right), u\left(t_{0}\right), y\left(t_{0}\right), \ldots,\right. \\
\left.y^{(k)}\left(t_{0}\right), \bar{v}_{k}\left(t_{0}\right)\right)
\end{gathered}
$$

when $\varphi=\left(r_{1}^{\left(\alpha_{1}\right)}, \ldots, r_{m}^{\left(\alpha_{m}\right)}\right)^{T}, \quad \xi_{i}\left(t_{0}\right)=$ $\left(r_{i}^{\left(\beta_{i}\right)}\left(t_{0}\right), \ldots, r_{i}^{\left(\alpha_{i}-1\right)}\left(t_{0}\right)\right)^{T}(i=1, \ldots, m)$, we have output of nonlinear DAE subsystems satisfies $y_{i}^{\left(\alpha_{i}\right)}(t)=\varphi_{i}(t), i=$ $1, \ldots, m$ (where $\varphi$ is new control input to be designed). By Definition 3, systems (18) are $\alpha$-order integral right inverse systems realized by state-feedback and dynamic compensation. This completes the proof.

There are two possible difficulties existing in realization of inverse systems: The one hand, the proposed method depends on the exact model of the controlled systems and sensitive to the perturbation of parameters or variation of structure. On the other hand, analysis solution of manipulated control 
may be difficult to be obtained. Inspired by [16], we may use the excellent approximation ability of Neural Networks to overcome the imprecise model and unknown analysis solution of manipulated control. This will be the next work we will undertake.

\section{Decentralized Excitation and Valve Coordinative Control of Synchronous Generator}

Excitation and valve coordinative control is studied for one synchronous generator based on the scheme proposed in this paper. For the sake of simplicity, the subscript $i$ is still omitted.

The mathematical model of synchronous generator excitation and valve coordinative control is described by the following two-input two-output nonlinear DAE subsystems [10]:

$$
\begin{gathered}
\dot{\delta}=f_{1}(\cdot)=\omega-\omega_{0} \\
\dot{\omega}=f_{2}(\cdot)=\frac{\omega_{0}}{H}\left\{P_{H}+C_{M L} P_{m 0}-\frac{D}{\omega_{0}}\left(\omega-\omega_{0}\right)\right. \\
\left.-\left[E_{q}^{\prime}+\left(x_{q}-x_{d}^{\prime}\right) I_{d}\right] I_{q}\right\} \\
\dot{E}_{q}^{\prime}=f_{3}(\cdot)=\frac{1}{T_{d 0}^{\prime}}\left[E_{f}-E_{q}^{\prime}-\left(x_{q}-x_{d}^{\prime}\right) I_{d}\right] \\
\dot{P}_{H}=f_{4}(\cdot)=\frac{1}{T_{H \Sigma}}\left(-P_{H}+C_{H} P_{m 0}+C_{H} U_{c}\right) \\
g(x, z, u, \bar{v})=0,
\end{gathered}
$$

where

$$
\begin{aligned}
& g(x, z, u, \bar{v}) \\
& =\left(\begin{array}{c}
P_{t}-\left[E_{q}^{\prime}+\left(x_{q}-x_{d}^{\prime}\right) I_{d}\right] I_{q}+r_{a}\left(I_{d}^{2}+I_{q}^{2}\right) \\
\theta_{U}-\delta+\operatorname{arcctg} \frac{x_{q} I_{q}-r_{a} I_{d}}{E_{q}^{\prime}-x_{d}^{\prime} I_{d}-r_{a} I_{q}} \\
I_{t}-\sqrt{I_{d}^{2}+I_{q}^{2}} \\
Q_{t}-E_{q}^{\prime} I_{d}+x_{q} I_{q}^{2}+x_{d}^{\prime} I_{d}^{2}
\end{array}\right)
\end{aligned}
$$

where the differential variables $x=\left(\delta, \omega, E_{q}^{\prime}, P_{H}\right)^{T}$ are relative power angle between $\mathrm{G} 1$ and $\mathrm{G} 4$, rotate speed deviation of $\mathrm{G} 1$, $q$-axis transient potential, and the high pressure mechanical power, respectively, the algebraic variables $z=\left(P_{t}, \theta_{U}, I_{d}, I_{q}\right)^{T}$ are active power, the angle of voltage, the $d$-axis current, and the $q$-axis current, respectively, and the interconnection input $\bar{v}=\left(I_{t}, Q_{t}\right)^{T}$ are the generator stator current and the reactive power, respectively. The manipulated input $u=\left(E_{f}, U_{c}\right)^{T}$ are induction electromotive force and the governor position, respectively. The controlled output $y$ is chosen as voltage and rotate speed deviation, respectively:

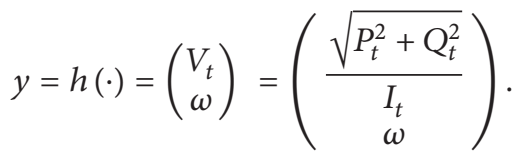

The others are the systems parameters.

The Jacobian matrix of $g(x, z, u, \bar{v})$ with respect to $z$ is

$$
\begin{aligned}
\frac{\partial g}{\partial z} & =\left(\begin{array}{cccc}
1 & 0 & \left(x_{q}-x_{d}^{\prime}\right) I_{q}+2 r_{a} I_{d} & \left(x_{q}-x_{d}^{\prime}\right) I_{d}+2 r_{a} I_{q} \\
0 & 1 & \frac{-1}{1+A^{2}} \frac{\partial A}{\partial I_{d}} & \frac{-1}{1+A^{2}} \frac{\partial A}{\partial I_{q}} \\
0 & 0 & \frac{I_{d}}{I_{t}} & -\frac{I_{q}}{I_{t}} \\
0 & 0 & -E_{q}^{\prime}+2 x_{d}^{\prime} I_{d} & 2 x_{q} I_{q}
\end{array}\right) \\
& \triangleq\left(\begin{array}{llll}
1 & 0 & a_{13} & a_{14} \\
0 & 1 & a_{23} & a_{24} \\
0 & 0 & a_{33} & a_{34} \\
0 & 0 & a_{43} & a_{44}
\end{array}\right),
\end{aligned}
$$

where $A=\left(x_{q} I_{q}-r_{a} I_{d}\right) /\left(E_{q}^{\prime}-x_{d}^{\prime} I_{d}-r_{a} I_{q}\right)$. It can be verified that under the normal operating condition, the following equation $\operatorname{det}(\partial g / \partial z)=-\left(1 / I_{t}\right)\left(2 x_{q} I_{d} I_{q}+E_{q}^{\prime} I_{q}-2 x_{d}^{\prime} I_{d} I_{q}\right) \neq 0$ holds, that is, matrix $\partial g / \partial z$ is of full rank and generator is of index one.

From (22) and (23), we can get the differential equation of $z$ as follows:

$$
\dot{z}=-\left(\frac{\partial g}{\partial z}\right)^{-1}\left(\frac{\partial g}{\partial x}\right) f-\left(\frac{\partial g}{\partial z}\right)^{-1}\left(\frac{\partial g}{\partial \bar{v}}\right) \dot{\bar{v}}
$$

where

$$
\begin{gathered}
\left(\frac{\partial g}{\partial z}\right)^{-1}=\left(\begin{array}{cccc}
1 & 0 & b_{1} & c_{1} \\
0 & 1 & b_{2} & c_{2} \\
0 & 0 & b_{3} & c_{3} \\
0 & 0 & -a_{33}^{-1} a_{43} & 1
\end{array}\right), \\
b_{1}=-a_{33}^{-1} a_{13}-a_{33}^{-1} a_{43} c_{1}, \quad b_{2}=-a_{33}^{-1} a_{23}-a_{33}^{-1} a_{43} c_{2}, \\
b_{3}=1-a_{33}^{-1} a_{43} c_{3}, \quad c_{1}=-c_{4}^{-1} a_{14}+a_{33}^{-1} c_{4}^{-1} a_{13} a_{34}, \\
c_{2}=-c_{4}^{-1} a_{24}+a_{33}^{-1} c_{4}^{-1} a_{23} a_{34}, \\
c_{3}=c_{4}^{-1} a_{34}, \quad c_{4}=a_{44}-a_{33}^{-1} a_{34} a_{43} .
\end{gathered}
$$




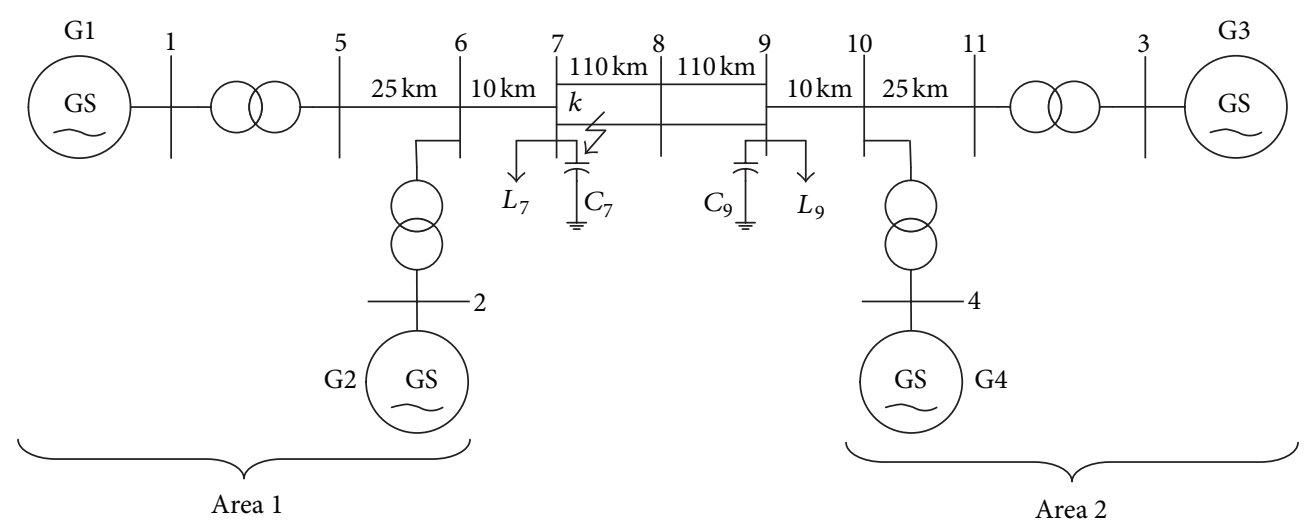

FIgURE 1: Two-area four-machine power system.

The Jacobian matrix of $g$ with respect to $x$ and interconnection input $\bar{v}$ are

$$
\begin{gathered}
\frac{\partial g}{\partial x}=\left(\begin{array}{cccc}
0 & 0 & -I_{q} & 0 \\
-1 & 0 & \frac{-1}{1+A^{2}} \frac{\partial A}{\partial E_{q}^{\prime}} & 0 \\
0 & 0 & 0 & 0 \\
0 & 0 & -I_{d} & 0
\end{array}\right), \\
\frac{\partial g}{\partial \bar{v}}=\left(\begin{array}{ll}
0 & 0 \\
0 & 0 \\
1 & 0 \\
0 & 1
\end{array}\right) .
\end{gathered}
$$

According to the recursive algorithm, the relative degree of (22) is 2 and the solution of $u$ can be derived as follows:

$$
\begin{aligned}
E_{f}= & \frac{T_{d_{0}}^{\prime}}{-I_{q}-c_{1} I_{d}} \\
& \times\left\{\frac{1}{P_{t}}\left[\frac{1}{I_{t}} \sqrt{P_{t}^{2}+Q_{t}^{2}}\left(I_{t}^{2} \dot{y}_{1}+\dot{I}_{t} \sqrt{P_{t}^{2}+Q_{t}^{2}}\right)-Q_{t} \dot{Q}_{t}\right]\right. \\
& \left.+b_{1} \dot{I}_{t}+c_{1} \dot{Q}_{t}\right\}+E_{q}^{\prime}+\left(x_{d}-x_{d}^{\prime}\right) I_{d}, \\
U_{c}= & \frac{\omega_{0} C_{H}}{H T_{H \Sigma}} \quad \\
& \times\left\{\ddot{y}_{2}-\frac{\omega_{0}}{H T_{H \Sigma}}\left(-P_{H}+C_{H} P_{m 0}\right)\right. \\
& \quad-\frac{D}{\omega_{0}} f_{2}-I_{q} f_{3}-\left(x_{q}-x_{d}^{\prime}\right) c_{3} I_{d} I_{q} f_{3} \\
& +\left(x_{q}-x_{d}^{\prime}\right) b_{3} I_{q} \dot{I}_{t}+\left(x_{q}-x_{d}^{\prime}\right) c_{3} I_{q} \dot{Q}_{t} \\
\quad & -E_{q}^{\prime} I_{d} f_{3}+a_{33}^{-1} a_{43} E_{q}^{\prime} \dot{I}_{t}+E_{q}^{\prime} \dot{Q}_{t}
\end{aligned}
$$

$$
\begin{aligned}
& -\left(x_{q}-x_{d}^{\prime}\right) I_{d}^{2} f_{3}+a_{33}^{-1} a_{43}\left(x_{q}-x_{d}^{\prime}\right) I_{d} \dot{I}_{i} \\
& \left.+\left(x_{q}-x_{d}^{\prime}\right) I_{d} \dot{Q}_{t}\right\} .
\end{aligned}
$$

According to $(18)$, the $(1,2)$-order integral right inverse systems for synchronous generator (22) can be constructed, with which the decoupling and linearization of the composed systems can be achieved.

The simulation is conducted based on a two-area fourmachine power systems (as shown in Figure 1).

The parameters of each generator and transformer are the same and the other parameters can be found in [17]. Systems operating condition is as follows: at first systems operate under double circuit stable state, then a three-phase symmetrical earth fault happens at $k$ point at line 7-8 on 0.5 second and the ground reactance of the failure point is 0.0001 pu.The fault is cut on 0.65 second and the systems return to original operating condition. Generators G1, G3 both adopted inverse excitation and valve controller and the closed-loop $\varphi_{1}, \varphi_{2}$ adopted PID controller where $\varphi_{1}$ only adopts proportion part equal to 10 and $\varphi_{2}$ adopt proportion part equal to 30 and differential part equal to 5 . The other generators adopt traditional linear controller. The simulation is based-on MATLAB and the results are shown in Figure 2.

As shown in Figure 2, when generators G1 and G3 both equipped excitation and valve coordinative inverse controller, both interarea oscillation and area oscillation are improved dramatically.

With noting that $x_{d}, x_{q}$ cannot be measured online. To overcome this difficulty, we can adopt the method proposed in [16] to replace the $x_{d}, x_{q}$ with local measured signals.

\section{Conclusion}

In this paper, the problem of invertibility for a special class of nonlinear DAE subsystems is studied. The definitions of inverse systems for nonlinear DAE subsystems are put forward. Then a recursive algorithm is given, with which to determine the invertibility of nonlinear DAE subsystems. Physically realizable right inverse systems are constructed 


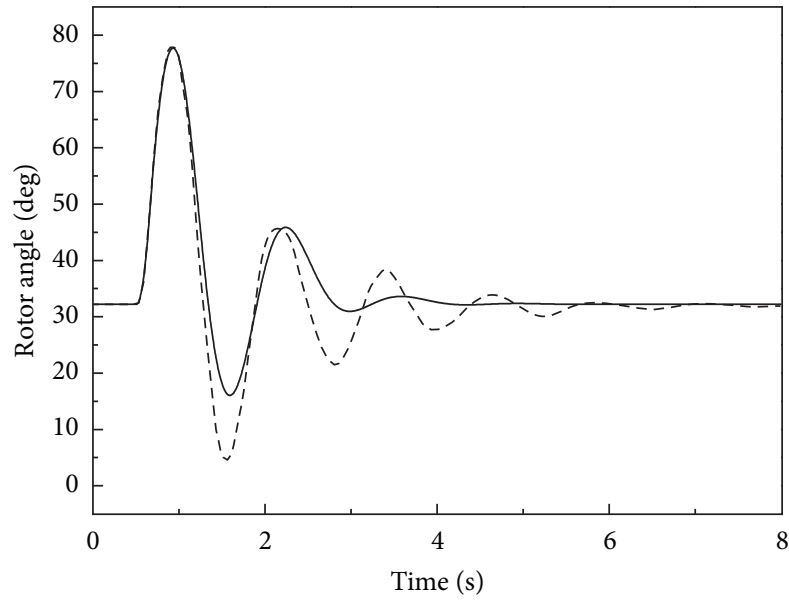

(a) Relative power angle between G1 and G4

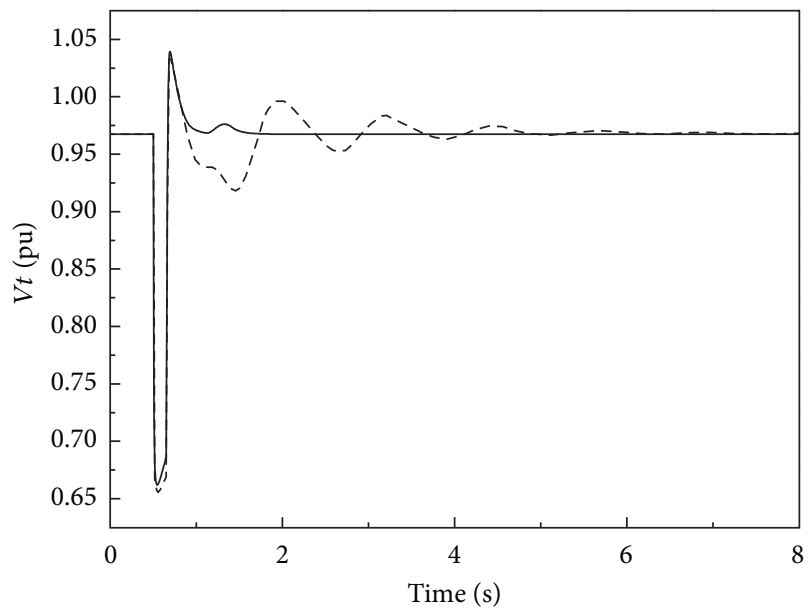

(c) Load bus voltage of G1

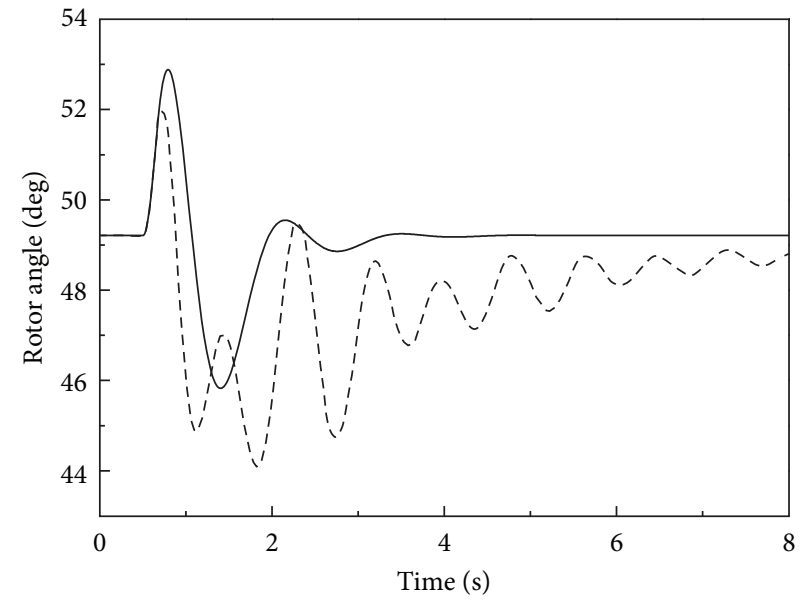

(b) Relative power angle between G3 and G4

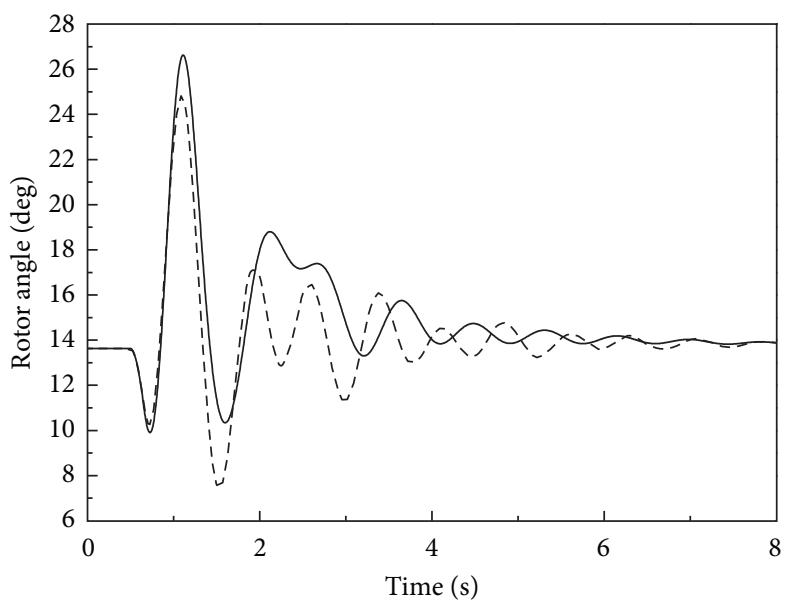

(d) Relative power angle between G1 and G2

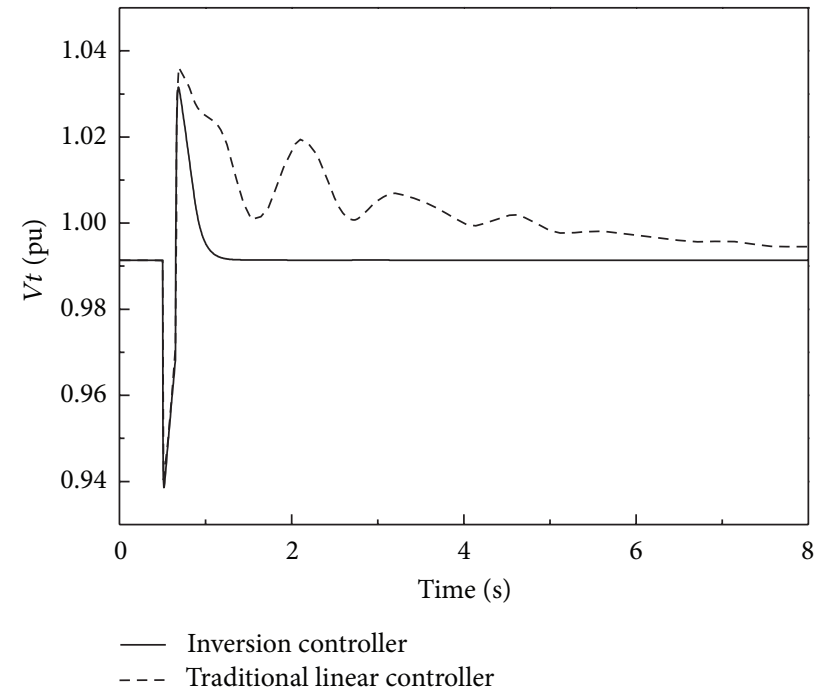

(e) Load bus voltage of G3

FIGURE 2: Simulation results of G1 and G3. 
through state-feedback and dynamic compensation, with which the decoupling and linearization of composite systems are achieved so that various linear control theorems and methods can be applied. Not restricted to power systems components decentralized control, the result of this paper is also meaningful to decentralized control of other areas of large-scale systems.

\section{Acknowledgments}

This work is supported by the National Natural Science Foundation of China (61004001, 61104103, 51177019), Natural Science Foundation of Jiangsu Province of China (BK2011826), Jiangsu Government Scholarship for Overseas Studies, Open Project of Key Laboratory of Measurement and Control of Complex Systems of Engineering, Ministry of Education, Southeast University (MCCSE2012A07), and State Grid Corporation of China, Major Projects on Planning and Operation Control of Large Scale Grid (SGCC-MPLG022-2012).

\section{References}

[1] S. L. Campbell, N. K. Nichols, and W. J. Terrell, "Duality, observability, and controllability for linear time-varying descriptor systems," Circuits Systems and Signal Processing, vol. 10, no. 4, pp. 455-470, 1991.

[2] D. Lin, W. Lan, and M. Li, "Composite nonlinear feedback control for linear singular systems with input saturation," Systems and Control Letters, vol. 60, no. 10, pp. 825-831, 2011.

[3] S. L. Sun and Y. Y. Chen, "Stabilization of a kind of nonlinear discrete singular large-scale control systems," Advance in Natural Science, vol. 5, no. 2, pp. 10-13, 2012.

[4] M. Darouach, L. Boutat-Baddas, and M. Zerrougui, " $H_{\infty}$ observers design for a class of nonlinear singular systems," Automatica, vol. 47, no. 11, pp. 2517-2525, 2011.

[5] R. K. Mandela, R. Rengaswamy, S. Narasimhan, and L. N. Sridhar, "Recursive state estimation techniques for nonlinear differential algebraic systems," Chemical Engineering Science, vol. 65 , no. 16, pp. 4548-4556, 2010.

[6] Q. Zang and X.-Z. Dai, "Output feedback stabilization control for nonlinear differential-algebraic equation systems," Acta Automatica Sinica, vol. 35, no. 9, pp. 1244-1248, 2009 (Chinese).

[7] L. Petzold, "Differential/algebraic equations are not ode's," SIAM Journal on Scientific and Statistical Computing, vol. 3, no. 3, pp. 367-384, 1982.

[8] D. J. Hill and I. M. Y. Mareels, "Stability theory for differential/algebraic systems with application to power systems," IEEE transactions on circuits and systems, vol. 37, no. 11, pp. 1416-1423, 1990.

[9] A. R. Ríos and M. L. López, "Order-reduction strategy of nonlinear differential-algebraic equation models with application on power systems," Electric Power Components \& Systems, vol. 40, no. 15, pp. 1690-1707, 2012.

[10] X.-Z. Dai and K.-F. Zhang, "Interface concept and structural model of complex power systems," Proceedings of the Chinese Society of Electrical Engineering, vol. 27, no. 7, pp. 7-12, 2007 (Chinese).

[11] X.-Z. Dai, K.-F. Zhang, and Q. Zang, "Nonlinear decentralized control method of power systems based on component structural model," Proceedings of the Chinese Society of Electrical Engineering, vol. 28, no. 22, pp. 15-22, 2008 (Chinese).
[12] M. T. Frye, Y. L. lu, and C. J. Qian, “Decentralized output feedback control of large-scale nonlinear systems interconnected by unmeasurable states," Proceeding of the American Control Conference, vol. 5, pp. 4267-4272, 2004.

[13] R. M. Hirschorn, "Invertibility of multivariable nonlinear control systems," IEEE Transactions on Automatic Control, vol. 24, no. 6, pp. 855-865, 1979.

[14] S. Tan and J. Vandewalle, "Inversion of singular systems," IEEE transactions on circuits and systems, vol. 35, no. 5, pp. 583-587, 1988.

[15] M. El-Tohami, V. Lovass-Nagy, and P. D. L. Powers D. L., "On minimal order inverse of discrete-time descriptor systems," International Journal of Control, vol. 41, no. 4, pp. 991-1004, 1985.

[16] X. Dai, D. He, T. Zhang, and K. Zhang, "ANN generalised inversion for the linearisation and decoupling control of nonlinear systems," IEE Proceedings, vol. 150, no. 3, pp. 267-277, 2003.

[17] P. Kundur, Power System Stability and Control, McGraw-Hill, 1994. 


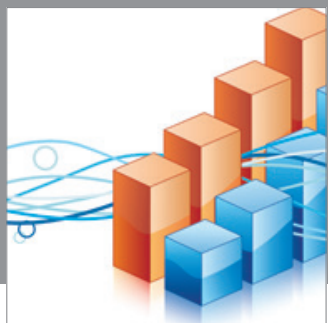

Advances in

Operations Research

mansans

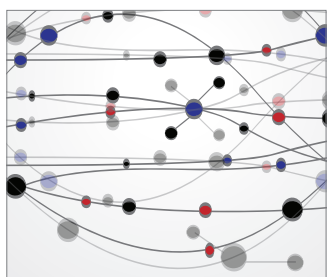

The Scientific World Journal
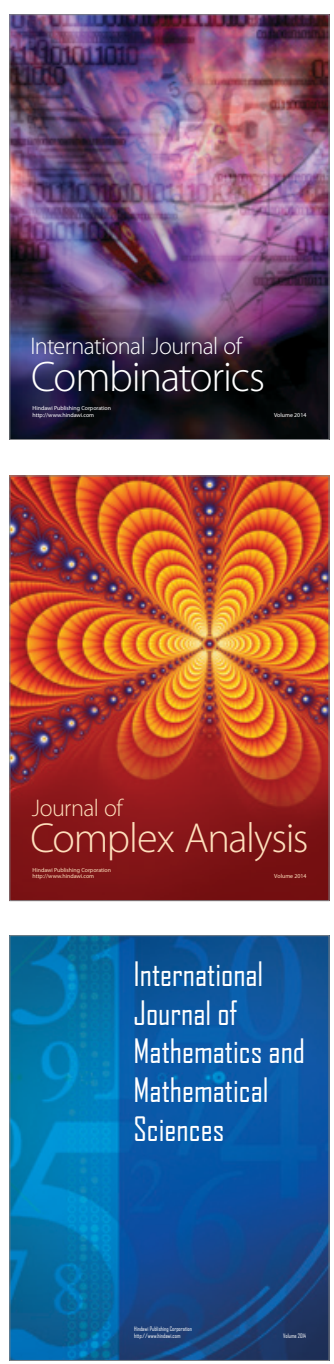
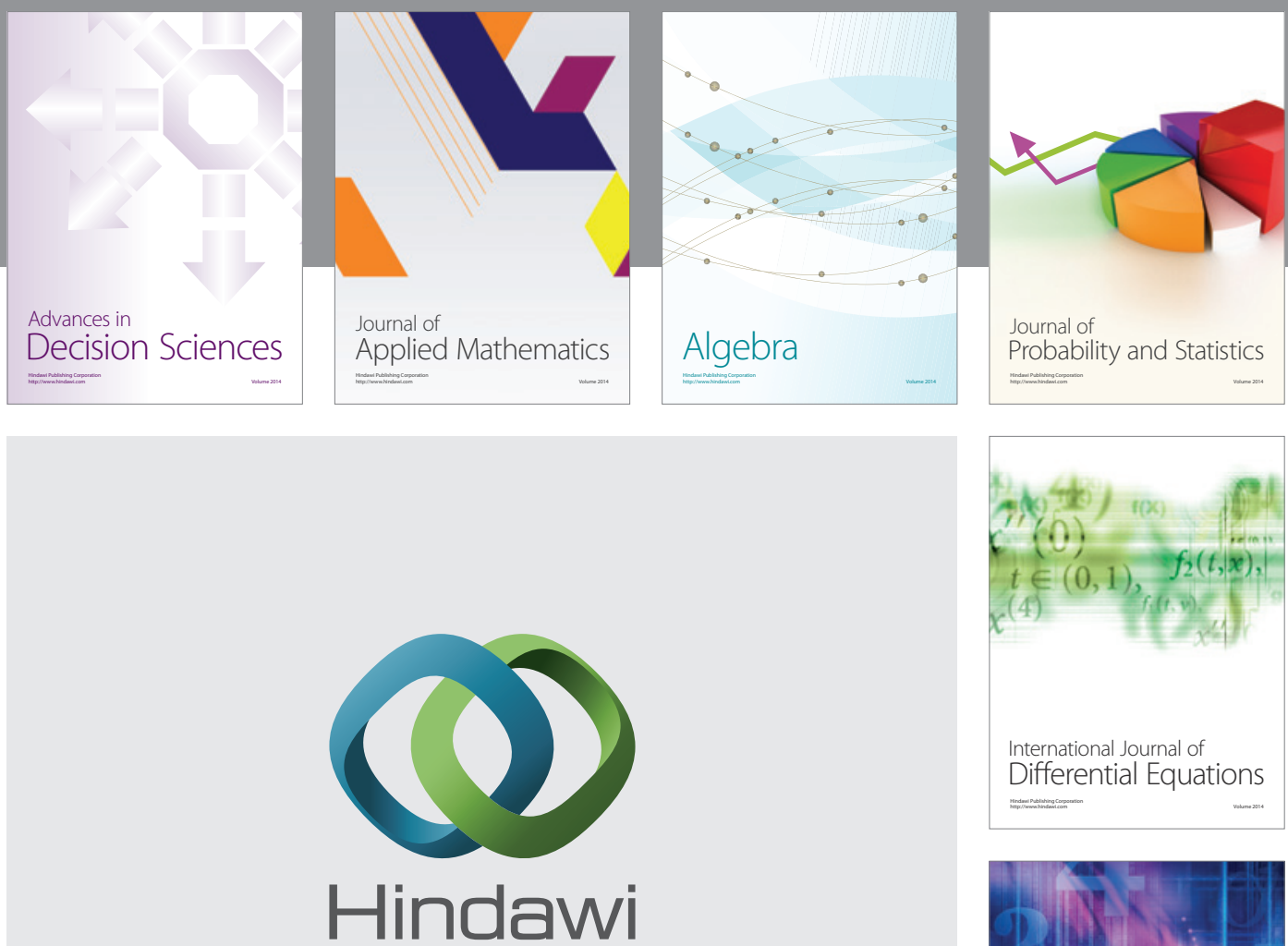

Submit your manuscripts at http://www.hindawi.com
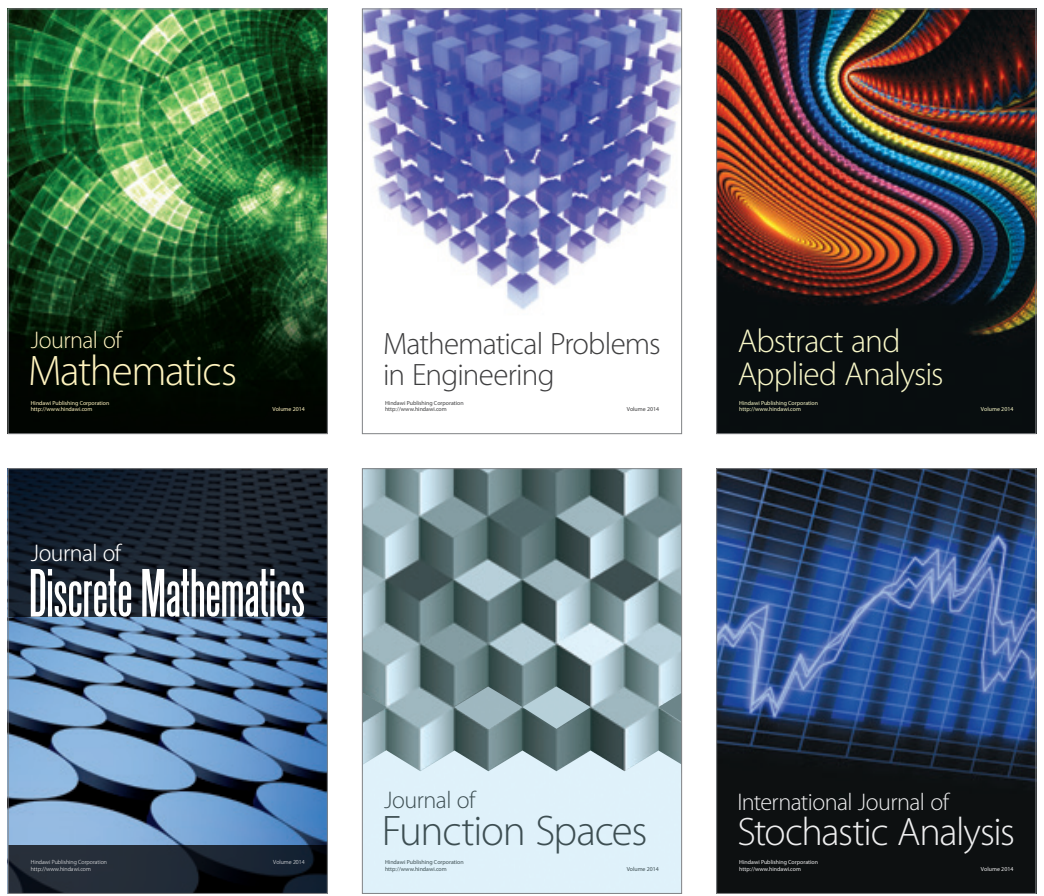

Journal of

Function Spaces

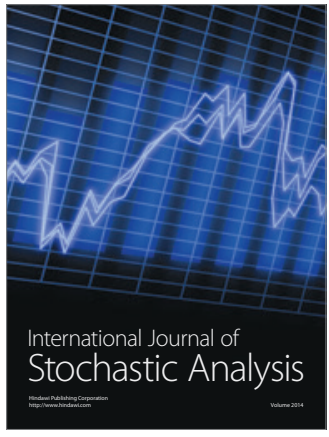

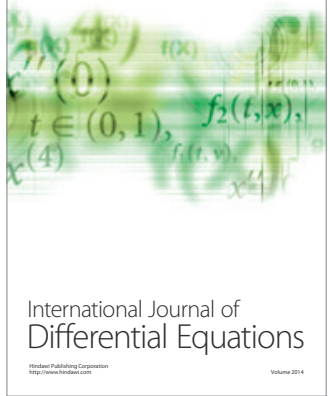
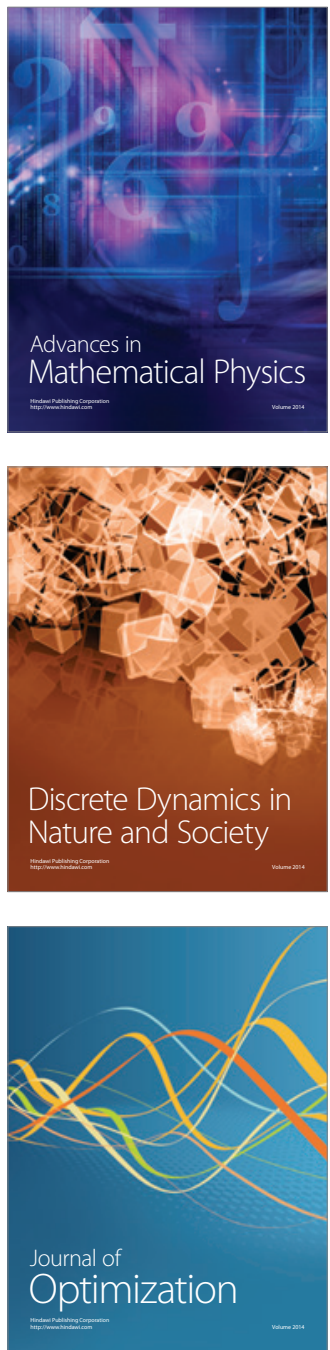